\title{
Post-Transplant Diabetes Mellitus in Renal Allograft Recipients: A Matched-Pair Control Study
}

\author{
A. von Kiparski ${ }^{1}$, D. Frei ${ }^{1}$, G. Uhlschmid ${ }^{2}$, F. Largiadèr ${ }^{2}$ and U. Binswanger ${ }^{1}$ \\ ${ }^{1}$ Nephrology Section, Department of Medicine; and ${ }^{2}$ Department of Surgery, University Hospital, Zurich, Switzerland
}

\begin{abstract}
The incidence of post-transplant diabetes mellitus was evaluated retrospectively in 901 consecutive renal transplant recipients. Thirty-two $(3.6 \%)$ patients developed diabetes mellitus requiring drug therapy. 18 of 32 became hyperglycaemic within 3 months of transplantation. Post-transplant diabetes mellitus occurred in 24 of $628(3.8 \%)$ patients treated with conventional therapy consisting in azathioprine and prednisone, and in 8 of 273 (2.9\%) patients receiving cyclosporin $\mathrm{A}(\mathrm{CsA})$ in addition (triple therapy). To identify predisposing factors 32 nondiabetic patients matched for age, sex, number of graft, immunosuppressive protocol, and graft function at onset of diabetes were used as case controls. Thirteen of 32 patients with diabetes mellitus and 5 of 32 control patients had abnormal glucose tolerance pretransplant $(P<0.025)$. HLA-B8 was significantly more frequent in patients with post-transplant diabetes mellitus than in control patients ( 9 of 29 vs 2 of $31, P<0.02$ ). Twelve $(38 \%)$ patients became diabetic during or immediately after anti-rejection therapy with intravenous pulse prednisone. Four diabetic patients experienced chronic pancreatitis pre-transplant. Family history of diabetes mellitus, bodyweight, number of rejection episodes, and immunosuppressive drug doses were similar in both groups. Actuarial patient and graft survival was not significantly different in diabetic patients and controls, although 10-year data tended to be better in controls. Thus, post-transplant diabetes mellitus was not a frequent complication in patients sometimes predisposed by
\end{abstract}

Correspondence and offprint requests to: Dr D. Frei, Nephrology Section, Department of Internal Medicine, University Hospital, Rämistr. 100, 8091 Zurich, Switzerland. an impaired glucose tolerance pre-transplant and was triggered by pulse prednisone therapy in $38 \%$.

Key words: Cyclosporin; Diabetes mellitus; Prednisone; Renal transplantation

\section{Introduction}

Persistent hyperglycaemia has been repeatedly observed in renal allograft recipients and is believed to be induced by immunosuppressive therapy. In 1964 Starzl [1] described the appearance of 'steroid diabetes' in previously nondiabetic renal transplant recipients. This was confirmed by several other groups [2-6]. Some authors observed post-transplant diabetes mellitus in association with antilymphocyte globulin [7] or antithymocyte globulin therapy [8]. Depending on the criteria of definition for hyperglycaemia, the incidence of post-transplant diabetes mellitus varied from $3 \%$ to $46 \%$ in these studies. Recently a greater frequency has been reported in renal allograft recipients receiving azathioprine and CsA compared to conventional protocols with azathioprine and prednisone [9-11]. The pathogenesis is not completely understood and diabetogenic risk factors other than immunosuppression were not clearly identified. The aim of this study was to determine the frequency of post-transplant diabetes mellitus requiring drug therapy in a large population of kidney transplant recipients treated with triple therapy and conventional therapy. To evaluate predisposing and triggering factors patients developing post-transplant diabetes mellitus were compared to matched controls. 


\section{Patients and Methods}

\section{Patients}

We retrospectively reviewed the course of 1149 consecutive cadaveric renal transplants performed in 986 patients at the University Hospital of Zurich between December 1964 and August 1988. Of the recipients, 901 were not diabetic prior to transplantation. Post-transplant diabetes mellitus was defined as follows: (1) two consecutive fasting blood glucose values $>6.7 \mathrm{mmol} / \mathrm{l}$, and (2) administration of insulin or oral hypoglycaemic drugs. According to these criteria 32 patients were included in the study group. Thirty-two non-diabetic recipients matched for age ( \pm 5 years), sex, immunosuppressive therapy, number of transplant, and graft function were selected as case controls to determine risk factors and survival rates. The time of transplantation was as similar as possible compared to the study 'group ( \pm 3 years). Each control was analysed at the time when the matched patient manifested diabetes mellitus and graft function was considered equal if serum creatinine values were in the same range according to the following classification: $<130,130-260$, $260-400,>400 \mu \mathrm{mol} / \mathrm{l}$. The clinical course of diabetic patients and controls was followed until August 1988 unless the patient died earlier.

The cadaveric allografts in both groups were selected on the basis of HLA typing and a negative cross-match between donor lymphocytes and recipients' sera. Three diabetic patients and one control were transplanted before 1969 without HLA typing results. The number of HLA-A and B antigen mismatches averaged $0.55 \pm 0.63$ (SD) and $1.10 \pm 0.62$ in the study group compared to $0.87 \pm 0.62$ and $1.17 \pm 0.75$ in controls. Since 1979, HLA-DR typing has been performed additionally in 15 diabetic patients and 20 controls (mean number of DR mismatches $0.53 \pm 0.64$ and $0.65 \pm 0.59$, respectively). The number of HLA antigens described to be associated positively (HLA-B8, B15, DR3, DR4) or negatively (HLA-B7, DR2) with insulin-dependent diabetes mellitus $[12,13]$ was calculated in both groups.

\section{Immunosuppression}

Since 1984, 273 recipients received triple therapy. CsA was given preoperatively, and on the first 3 postoperative days $3 \mathrm{mg} / \mathrm{kg}$ per day intravenously. The oral medication was started on the 4 th day with $5 \mathrm{mg} / \mathrm{kg}$ per day. The dosage was adjusted to maintain a 24 -h trough level of $300-500 \mathrm{ng} / \mathrm{ml}$ during the first 3 months and of 200 $400 \mathrm{ng} / \mathrm{ml}$ in the following, as measured by whole blood fluorescence polarisation immunoassay (Abbott, North Chicago, USA). Azathioprine was given postoperatively in an oral dose of $1 \mathrm{mg} / \mathrm{kg}$ per day. Oral prednisone was administered at a dose of $1 \mathrm{mg} / \mathrm{kg}$ per day followed by $40 \mathrm{mg} /$ day from the $3 \mathrm{rd}$ week post-transplant. Thereafter, the dose was continuously reduced by $10 \mathrm{mg}$ monthly to a maintenance dose of $10 \mathrm{mg} /$ day. The cumulative prednisone dose after 1 year was $5.7 \mathrm{~g}$. Six hundred and twenty-eight patients were treated with conventional therapy (1964-1984) consisting of azathioprine $3 \mathrm{mg} / \mathrm{kg}$ per day plus prednisone as described above.

\section{Rejection Episodes}

Rejection episodes were treated with steroid pulses (methylprednisolone $1 \mathrm{~g} /$ day i.v. for 3-5 days) or, if prednisone-resistant, with antithymocyte globulin $(3 \mathrm{mg} / \mathrm{kg}$ per day) or OKT3 $(5 \mathrm{mg} /$ day $)$ for 7-14 days. The diagnosis of rejection episodes was based upon clinical criteria (fever, graft swelling, tenderness, oliguria), a significant increase in serum creatinine ( $20 \%$ or more), normal or subtherapeutic CsA blood values, and characteristic graft biopsy in the case of episodes resistant to steroid pulses.

\section{Oral Glucose Tolerance Test}

An oral glucose tolerance test (GTT) prior to transplantation was performed in 21 of 32 patients with posttransplant diabetes mellitus and in 23 of 32 controls. The remaining recipients were analysed on the basis of their fasting serum blood glucose values, except for one diabetic and one control of whom the pretransplant blood glucose values were not known. The GTT results were subdivided as follows: (1) diabetic type with fasting blood glucose $>6.7 \mathrm{mmol} / \mathrm{l}$ and/or 2-h blood glucose $>10 \mathrm{mmol} / \mathrm{l}$; (2) normal type with fasting blood glucose $\leq 5.8 \mathrm{mmol} / \mathrm{l}$ and/or $1-\mathrm{h}$ blood glucose $\leq 10.0 \mathrm{mmol}$ and 2-h blood glucose $\leq 6.7 \mathrm{mmol} / \mathrm{l}$; and (3) impaired glucose tolerance with fasting blood glucose between 5.8 and $6.7 \mathrm{mmol} / 1$ and/or 1 -h blood glucose $>10.0 \mathrm{mmol} / 1$ and 2-h blood glucose between 6.7 and $10.0 \mathrm{mmol} / \mathrm{l}$.

\section{Data Analysis}

Various clinical and laboratory parameters were tabulated and compared in control and study recipients. Student's $t$ test and Fisher's exact test were employed to test significance of results. Cumulative actuarial patient and graft survival was calculated by the life table method [14].

\section{Results}

Table 1 shows the matching criteria of diabetic patients and controls. One diabetic could not be matched for sex and another diabetic with a third graft was matched with a first-graft recipient. Twenty-seven diabetic patients and 
28 controls were first-graft recipients. In each group conventional therapy was administered to 24 and triple therapy to eight patients. Post-transplant diabetes mellitus occurred in $32(3.6 \%)$ of 901 kidney transplant recipients and was not more frequent in patients receiving triple therapy compared to conventional therapy (Table $2)$. Twenty $(63 \%)$ diabetic patients required insulin therapy with a mean dose of $35.6 \pm 13.3$ units per day at the onset of diabetes. Only three of these 20 were less than 30 years old and could be theoretically classified as type I diabetes mellitus. Eighteen $(56 \%)$ recipients became hyperglycaemic in the first 3 months after transplantation (mean $42.6 \pm 20.5$ days post-transplant). The remaining 14 patients developed diabetes mellitus from day 300 up to 13 years after transplantation. The duration of diabetes varied from 1 month to 13 years and 24 of 32 recipients had post-transplant diabetes mellitus for more than 1 year.

Table 1. Matching criteria of patients with post-transplant diabetes mellitus (PTDM) and controls

\begin{tabular}{|c|c|c|}
\hline Matching criteria & $\begin{array}{l}\text { PTDM } \\
(n=32)\end{array}$ & $\begin{array}{l}\text { Controls } \\
(n=32)\end{array}$ \\
\hline $\begin{array}{l}\text { Age at transplantation (years) } \\
\text { (Age range) }\end{array}$ & $\begin{array}{l}45.1 \pm 12.6^{1} \\
(22-65)\end{array}$ & $\begin{array}{l}45.5 \pm 11.8^{1} \\
(22-62)\end{array}$ \\
\hline Sex (male:female) & $22: 10$ & $21: 11$ \\
\hline 1st:2nd:3rd:4th graft & $27: 3: 1: 1$ & $28: 3: 0: 1$ \\
\hline $\begin{array}{l}\text { Serum creatinine at onset of } \\
\text { PTDM }(\mu \mathrm{mol} / 1)\end{array}$ & $141.3 \pm 123.2^{1}$ & $137.0 \pm 69.2^{1}$ \\
\hline CT:TT & $24: 8$ & $24: 8$ \\
\hline
\end{tabular}

${ }^{1}$ Mean $\pm \mathrm{SD} ; \mathrm{CT}$, conventional therapy; TT, triple therapy

Table 2. Frequency of post-transplant diabetes mellitus in renal transplant recipients according to immunosuppression

\begin{tabular}{llll}
\hline & $\begin{array}{l}\text { TT } \\
\text { (CsA, Aza, Pred) } \\
n=273\end{array}$ & $\begin{array}{l}\text { CT } \\
\text { (Aza, Pred) } \\
n=628\end{array}$ & $\begin{array}{l}\text { Total } \\
n=901\end{array}$ \\
\hline $\begin{array}{l}\text { Insulin therapy } \\
\begin{array}{l}\text { Oral antidiabetic } \\
\text { drugs }\end{array}\end{array}$ & 5 & 15 & 20 \\
\begin{tabular}{l} 
Total \\
\hline
\end{tabular} & 3 & 9 & 12 \\
\hline
\end{tabular}

TT, triple therapy; CT, conventional therapy

To evaluate predisposing factors several clinical variables were analysed both in patients with post-transplant diabetes mellitus and in controls (Table 3). In both groups, familial history of diabetes and bodyweight at transplantation were similar. Impaired glucose tolerance as measured by GTT and/or several consecutive fasting blood glucose values prior to transplantation were observed in $13(42 \%)$ diabetics and five controls $(P<0.025)$. Four patients with post-transplant diabetes mellitus suffered from chronic pancreatitis, with pancreas surgery in their history. In three of four cases exocrine organ insufficiency was diagnosed by reduced chymotrypsin excretion in the stool. One control experienced acute pancreatitis. HLA-B8 was significantly more frequent in diabetic patients than in controls ( 9 of 29 vs 2 of 31 ; $P<0.02)$ whereas HLA-A28 was only present in 2 of 29 diabetic recipients compared to 4 of 31 controls (Table 4.)

Table 3. Preoperative variables in patients with post-transplant diabetes mellitus (PTDM) and controls

\begin{tabular}{lll}
\hline & \multicolumn{2}{l}{ No. positive/No. tested } \\
\cline { 2 - 3 } & $\begin{array}{l}\text { PTDM } \\
(n=32)\end{array}$ & $\begin{array}{l}\text { Controls } \\
(n=32)\end{array}$ \\
\hline $\begin{array}{l}\text { Diabetes in family } \\
\text { Bodyweight }(\mathrm{kg}) \text { at } \\
\text { transplantation }\end{array}$ & $4 / 27$ & $3 / 24$ \\
$\begin{array}{l}\text { Impaired glucose tolerance } \\
\text { pretransplant (GTT) })^{3}\end{array}$ & $59.9 \pm 12.0^{1}$ & $57.94 \pm 10.0^{1}$ \\
\begin{tabular}{l} 
Pancreatitis pretransplant \\
\hline
\end{tabular} & $13 / 31^{2}$ & $5 / 31$ \\
\hline
\end{tabular}

${ }^{1}$ Mean \pm SD

${ }^{2} P<0.025$ vs controls

${ }^{3}$ GTT was performed in 21 diabetics and 23 controls. In the remaining patients impaired glucose tolerance was diagnosed by fasting blood glucose values except in one diabetic and one control

Table 4. Diabetes-associated HLA antigens in patients with posttransplant diabetes mellitus (PTDM) and controls

\begin{tabular}{lll}
\hline HLA antigens & \multicolumn{2}{l}{ No. positive/No. tested } \\
\cline { 2 - 3 } & $\begin{array}{l}\text { PTDM } \\
(n=32)\end{array}$ & $\begin{array}{l}\text { Controls } \\
(n=32)\end{array}$ \\
\hline Positive association with diabetes mellitus & & \\
HLA-A28 & $2 / 29$ & $4 / 31$ \\
B8 & $9 / 29^{1}$ & $2 / 31$ \\
B15 & $5 / 29$ & $3 / 31$ \\
DR3 & $2 / 16$ & $3 / 20$ \\
DR4 & $7 / 16$ & $2 / 20$ \\
Negative association with diabetes mellitus & \\
HLA-B7 & $5 / 29$ & $8 / 31$ \\
DR2 & $3 / 16$ & $6 / 20$ \\
\hline
\end{tabular}

${ }^{1} P<0.02$ vs controls

At the time of diagnosis the mean fasting blood glucose value of the study group was $15.9 \pm 7.7 \mathrm{mmol} / \mathrm{l}$ compared to $5.2 \pm 1.2 \mathrm{mmol} / 1$ in controls $(P<0.001)$. There was a correlation between anti-rejection therapy with pulse prednisone and the onset of post-transplant diabetes mellitus. Twelve of thirty-two (38\%) patients of the study group became diabetic during or immediately after pulse 
Table 5. Immunosuppressive therapy of diabetics and controls at onset of post-transplant diabetes mellitus $(\text { PTDM })^{1}$

\begin{tabular}{lllllll}
\hline & PTDM & & & \multicolumn{1}{l}{ Controls } \\
\cline { 2 - 3 } \cline { 5 - 6 } & CT $(n=24)$ & TT $(n=8)$ & & CT $(n=24)$ & TT $(n=8)$ \\
\hline Aza (mg/day) & $144.9 \pm 49.2$ & $55.4 \pm 22.4$ & & $129.7 \pm 47.0$ & $64.3 \pm 24.4$ \\
Pred (mg/day) & $26.5 \pm 17.0$ & $27.5 \pm 11.6$ & & $21.8 \pm 14.9$ & $27.1 \pm 9.5$ \\
Total dose of Pred (g) & $14.6 \pm 14.4$ & $8.4 \pm 9.1$ & & $17.7 \pm 15.2$ & $8.0 \pm 4.6$ \\
CsA (mg/day) & - & $282.5 \pm 87.8$ & & - & $360.0 \pm 152.3$ \\
CsA blood level (ng/ml) & - & $341.7 \pm 195.3$ & & - & $355.3 \pm 200.0$ \\
\hline
\end{tabular}

${ }^{1}$ Drug doses are expressed as mean values $\pm \mathrm{SD} ;{ }^{2}$ cumulative dose from transplantation to onset of PTDM. Aza, azathioprine; Pred, prednisone; CT, conventional therapy; TT, triple therapy

prednisone therapy. However, the frequency of rejection episodes from transplantation to onset of diabetes was similar in both groups. Eighteen of thirty-two patients with diabetes experienced 25 rejection episodes and 21 of 32 controls had 28 rejection episodes. The basic immunosuppression at onset of post-transplant diabetes mellitus is compared in Table 5 . In both groups the doses of prednisone (daily and cumulative from transplantation to onset of post-transplant diabetes mellitus, azathioprine and CsA were similar. Elevated CsA blood values were observed in two patients with post-transplant diabetes mellitus (605 and $640 \mathrm{ng} / \mathrm{ml}$ ) and in one control (736 ng/ml).

Patients with a late onset of post-transplant diabetes mellitus ( 300 days to 13 years, $n=14$ ) differed in some aspects from patients who developed diabetes mellitus early after transplant $(42.6 \pm 20.5$ days, $n=18)$ : only 3 of 14 'late' diabetic patients had an impaired glucose tolerance pretransplant as measured by GTT and/or several consecutive fasting blood glucose levels compared to 10 of 18 patients with 'early' post-transplant diabetes mellitus $(P=$ n.s. $)$. The onset of diabetes mellitus was not clearly associated with pulse prednisone therapy (2 of 14 'late' diabetics and 10 of 18 or $56 \%$ 'early' diabetics developed post-transplant diabetes mellitus during or immediately after anti-rejection therapy with pulse prednisone; $P<0.02)$. Moreover, HLA-B8 was present in 1 of 12 'late' diabetics compared to 8 of 17 patients with 'early' posttransplant diabetes mellitus $(P<0.05)$. Three of four patients with chronic pancreatitis pretransplant had a late onset of post-transplant diabetes mellitus.

During follow-up, $12(38 \%)$ diabetic patients and six $(19 \%)$ controls had to be hospitalised because of serious infections. The most common causes for hospitalisation were CMV infection $(n=4)$, erysipelas $(n=3)$ and abscesses $(n=3)$. Other reasons were sepsis, meningitis, pneumonia, and urinary tract infections. Cardiovascular complications such as myocardial infarction $(n=2)$, coronary heart disease $(n=5)$ or arteriosclerosis obliterans $(n=6)$ occurred in 9 of 32 patients with post-transplant diabetes mellitus and in 3 of 32 controls $(P=$ n.s. $)$. Patient and graft survival did not differ between the two groups 1 , 2 and 5 years post-transplant. However, long-term graft and patient survival were better for controls than for diabetic patients though not significantly. At 10 years, patient survival was $93 \%$ in controls ( $n$ at risk, 11 ) and $51 \%$ in diabetic patients ( $n$ at risk, 7 ) and graft survival was $85 \%$ in controls ( $n$ at risk, 11 ) compared to $54 \%$ in patients with post-transplant diabetes mellitus ( $n$ at risk, 8). Eleven diabetics and three controls died during follow-up; the main causes of death in patients with post-transplant diabetes mellitus were cardiovascular problems such as stroke $(n=2)$, myocardial infarction $(n=1)$ pulmonary thromboembolism $(n=1)$ or cardiopulmonary failure $(n=3)$. Other reasons were sepsis $(n=2)$, liver carcinoma $(n=1)$, and gastrointestinal bleeding $(n=1)$. The causes of death in controls were uraemia (patient refused dialysis), renal pelvis carcinoma, and cardiopulmonary failure.

\section{Discussion}

Post-transplant diabetes mellitus has been described in $3 \%-16 \%$ of renal allograft recipients [3-11] and one author [2] even reported occurrence in 67 of 145 patients. Most of the literature included patients with transient hyperglycaemia and/or without antidiabetic therapy; therefore, the incidence of post-transplant diabetes mellitus was higher than in the present series. Compared to the prevalence of diabetes mellitus of $1 \%-2 \%$ in a normal population [15], post-transplant diabetes mellitus requiring drug therapy was not a frequent complication in our patients. Nevertheless, Gunnarsson et al [2] and Yoshimura et al [10] noticed a high frequency of insulindependent diabetes mellitus of $18 \%$ and $14 \%$ respectively. In both reports greater doses of steroids than in the present study were administered during the first months post-transplant. The lowest incidence of $3 \%$ was observed by McGeown et al [4], who administered a low-dose steroid regimen ( $20 \mathrm{mg} /$ day prednisone) early post-transplant. Since CsA has been introduced 
in the immunosuppressive protocols following kidney transplantation, Gunnarsson et al [16] have noted a deterioration in the glucose metabolism of combined kidney and pancreas allograft recipients who had azathioprine replaced by CsA. This diabetogenic effect of CsA is thought to be dose-dependent and reversible [17]. Subsequently, two Japanese groups $[9,10]$ have reported a higher frequency of post-transplant diabetes mellitus in kidney allograft recipients treated with CsA and prednisone compared to conventional therapy and azathioprine and prednisone $(17 \%$ vs $13 \%$ and $8 \%$ vs $5 \%$ ). This was recently confirmed by Roth et al [11].

In our series, a higher frequency of post-transplant diabetes mellitus in patients treated with CsA as part of triple therapy compared to conventional therapy could not be observed. This may be due to the fact that patients with triple therapy were treated with smaller doses of CsA than in the studies cited above, especially early posttransplant. On the other hand, patients receiving triple therapy were compared to conventionally treated patients transplanted before 1984. Therefore, the follow-up of the conventional therapy group was longer and it might be possible that some patients with triple therapy will develop post-transplant diabetes mellitus later.

To answer the question why some recipients develop diabetes during immunosuppressive therapy while others do not, possible predisposing and triggering factors were examined in the literature. Gunnarsson et al [2] noted a significant correlation between the development of posttransplant diabetes mellitus and the total dose of prednisone administered or the frequency of rejection episodes. The work of others [3,5-7] has not confirmed these findings. In spite of the similar frequency of rejection episodes in the study and the control group we found a clear correlation between anti-rejection therapy with steroid pulses and the manifestation of post-transplant diabetes mellitus in $38 \%$ of the diabetic patients, suggesting that prednisone pulses are a triggering factor in otherwise predisposed patients.

David et al [3] proposed a genetic predisposition, reporting a significant correlation between the presence of HLA-A28 and the occurrence of post-transplant diabetes mellitus. No connections between HLA antigens described as associated positively (HLA-B8, B15, DR3, DR4) or negatively (HLA-B7, DR2) with insulindependent diabetes $[12,13]$ and post-transplant diabetes mellitus have been reported in the literature. In our series, HLA-B8 was significantly more frequent in diabetic patients than in controls although only three patients might be classified as having type I diabetes mellitus. The association of HLA-B8 and insulin-dependent diabetes mellitus has been shown to be secondary to an increase of HLA-DR3, and was explained by the known linkage disequilibrium between HLA-A, B, C and DR loci [18]. HLA-DR3 was not more frequent in diabetic patients compared to the controls. Testing for distribution of HLA antigens in a relatively small population may turn out to be significant merely by chance, and therefore the presence of HLA-B8 in patients with post-transplant diabetes mellitus cannot be clearly interpreted as a genetic risk factor.

In some previous reports the occurrence of posttransplant diabetes mellitus was related to familial history of diabetes, older age or greater bodyweight at transplantation $[2,7,8,11]$. In the present study, the patients were matched according to age and therefore the hypothesis of older age as a risk factor could not be evaluated. There was no significant difference in bodyweight between diabetic patients and controls. An impaired glucose tolerance pretransplant, as observed in almost $50 \%$ of our diabetics, could be a risk factor for the development of diabetes mellitus. However, Yoshimura et al [10] noted an abnormal GTT prior to transplantation in only $18 \%$. Chronic pancreatitis, as observed in four patients, might be a pathogenic factor depending on the degree of organ dysfunction.

In the present study, patients with late onset of posttransplant diabetes mellitus were included to evaluate whether they showed different clinical characteristics compared to the 'early' diabetics. Interestingly, only three of the 'late' diabetics were predisposed by an impaired glucose tolerance pretransplant as compared to 10 of 18 'early' diabetics. HLA-B8 was significantly more frequent in the latter group. The finding that only two 'late' diabetics developed post-transplant diabetes mellitus in association with pulse prednisone may be due to the fact that acute rejection episodes are more frequent in the early post-transplant period. It is possible that some patients with late onset of hyperglycaemia develop type II diabetes independently of immunosuppression with a similar frequency $(14$ of $901,1.6 \%)$ as the normal population [15]. Three of four patients with chronic pancreatitis pretransplant were 'late' diabetics, suggesting that in these patients the aggravation of pancreatitis rather than immunosuppression represents a pathogenic factor.

The influence of post-transplant diabetes mellitus on the long-term course of renal allograft recipients is not well known. Boudreaux et al [7] reported that such patients experienced significantly more infections requiring hospitalisation than did non-diabetic recipients. A similar trend was seen in the present series. Moreover, a tendency to cardiovascular complications could be observed in the patients with post-transplant diabetes mellitus. Ten-year graft and patient survival rates were lower in our study group than in controls, though not significantly. Gunnarsson et al [19] and Friedman et al [6] reported significantly poorer patient survival in diabetics than in controls at 1 (58\% vs $87 \%$ and $73 \%$ vs $83 \%$ respectively) and 2 years (44\% vs $77 \%$ and $67 \%$ vs $83 \%$ respectively). Infections were a frequent cause of death in 
the series of Friedman, as were cardiovascular reasons in our patients. Recently Roth et al [11] noted that actuarial graft survival was significantly less in patients with post-transplant diabetes mellitus at 3 and 4 years posttransplant. David et al [3] could not establish an unfavourable effect on either patient or transplant outcome.

In summary, persistent post-transplant diabetes mellitus requiring drug therapy was not a frequent complication in our series. An abnormal GTT prior to transplantation may identify predisposed patients. Pulse prednisone seems to be a triggering factor, especially in renal allograft recipients with an early onset of posttransplant diabetes mellitus.

\section{References}

1. Starzl TE. Experience in renal transplantation. Saunders, Philadelphia, 1964: 111-125

2. Gunnarsson R, Arner P, Lundgren G, Magnusson G, Oestman J, Groth CG. Diabetes mellitus-a more-common-than-believed complication of renal transplantation. Transplant Proc 1979; 11: 1280-1281

3. David DS, Cheigh JS, Braun DW, Fotino M, Stenzel KH, Rubin AL. HLA-A28 and steroid-induced diabetes in renal transplant patients. JAMA 1980; 243: 532-533

4. McGeown MG, Douglas JF, Brown WA et al, Advantages of low-dose steroid from the day after renal transplantation. Transplantation 1980; 29: 287-289

5. Dumler F, Hayashi H, Hunter J, Levin NW. Racial differences in the incidence of steroid diabetes in renal transplant patients. Henry Ford Hosp Med J 1982; 30: 14-16

6. Friedman EA, Shyh T, Beyer MM, Manis T, Butt KMH. Posttransplant diabetes in kidney transplant recipients. Am J Nephrol 1985; 5: 196-202
7. Boudreaux JP, McHugh L, Canafax DM et al. The impact of cyclosporine and combination immunosuppression on the incidence of posttransplant diabetes in renal allograft recipients. Transplantation 1987; 44: 376-381

8. Butt KMH, Parsa J, Emett L et al. Antithymocyte globulin-related diabetes in living related transplantation. Transplant Proc 1983; 15 : 1083-1085

9. Yagisawa T, Takahashi K, Teraska S, Toma H, Agishi T, Ota K. Deterioration in glucose metabolism in cyclosporine-treated kidney transplant recipients and rats. Transplant Proc 1986; 18: 1548-155I

10. Yoshimura N, Ichiro N, Yoshihiro Oet al. Effect of cyclosporine on the endocrine and exocrine pancreas in kidney transplant recipients. Am J Kidney Dis 1988; 12: 11-17

11. Roth D, Milgrom M, Esquenazi V, Fuller L, Burke G, Miller J. Posttransplant hyperglycemia-Increased incidence in cyclosporine-treated renal allograft recipients. Transplantation 1989; 47: 278-281

12. Cudworth AG, Festenstein H. HLA genetic heterogeneity in diabetes mellitus. Br Med Bull 1978; 34: 285-289

13. Jeannet M. Class II HLA antigens in autoimmune and immunemediated diseases. In: Solheim BG, Moller E, Ferrone S, eds. $H L A$ class II Antigens. Berlin, Heidelberg; Springer, 1986: 489-514

14. Cutler SJ, Ederer F. Maximum utilization of the life table method in analyzing survival. J. Chron Dis 1958; 8: 699-712

15. Assal JP, Liniger C, Froesch ER. Pathogenesis, etiology and epidemiology of diabetes mellitus. In: Labhart A, ed. Clinical Endocrinology. Berlin, Heidelberg; Springer, 1986: 766-770

16. Gunnarsson $R$, Klinthalm $G$, Lundgren $G$ et al. Deterioration in glucose metabolism in pancreatic transplant recipients after conversion from azathioprine to cyclosporine. Transplant Proc 1984; 16: 709-712

17. Engfeldt P, Tydén G, Gunnarsson R, Oestmann J, Groth CG. Impaired glucose tolerance with cyclosporine. Transplant Proc 1986; 18: 65-66

18. Sachs JA, Cudworth AG, Jaraquemada D, Gorsuch AN, Festenstein H. Type 1 diabetes and the HLA-D locus. Diabetologia $1980 ; 18: 41-43$

19. Gunnarsson R, Lundgren G, Magnusson G, Oest L, Groth CG. Steroid diabetes-a sign of overtreatment with steroids in the renal graft recipients? Scand J Urol Nephrol 1980; 54 [suppl]: 135-138

Received for publication 27.6 .89

Accepted in revised form 2.11 .89 